\author{
Турчанин М. А., Древаль Л. А., Агравал П. Г., Довбенко А. И., \\ Ильенко С. М.
}

\title{
НОВЫЕ НАПРАВЛЕНИЯ ТЕРМОДИНАМИЧЕСКИХ ИССЛЕДОВАНИЙ ВЫСОКОЭНТРОПИЙНЫХ СПЛАВОВ
}

Высокоэнтропийные сплавы (ВЭС) - это новый класс материалов, запатентованный в 2002 году Ин-Вей Ех в Тайване [1]. Главная идея патента заключалась в создании многокомпонентных материалов на базе металлических систем, содержащих более пяти основных компонентов, смешанных в приблизительно равных пропорциях. Результаты многих экспериментальных работ показывают, что ВЭС обладают высокими механическими и рабочими свойствами [2]. Для ВЭС не существует ограничений в выборе метода их получения. За последние двенадцать лет концепция ВЭС была значительно расширена исследовательскими группами из разных стран мира [2]. Интерес к данной теме растет с каждым годом.

ВЭС стали интересными в виду своих высоких механических и рабочих свойств. Огромное число работ, опубликованных в последнее время, направлены именно на изучение этих свойств и их взаимосвязь с микроструктурой ВЭС (типом фазы, набором фаз, механизмом их образования) [2,3]. Данная информация требует систематизации и обобщения для лучшего понимания и разработки данного класса материалов.

Для ВЭС можно говорить о следующей закономерности. Как правило, ВЭС, имеющие ОЦК структуру (ОЦК-ВЭС), характеризуются более высокими показателями твердости, прочности, износостойкости по сравнению с ВЭС с ГЦК структурой (ГЦК-ВЭС), при этом показатели износостойкости оказываются на уровне показателей для серого чугуна и нержавеющих сталей, а по прочности отдельные сплавы не уступают инконелям. В тоже время ГЦК-ВЭС характеризуются более высокими пластичностью (для отдельных представителей пластическая деформация может достигать 50 \%), деформационным упрочнением и сопротивлением усталости по сравнению с ОЦК-ВЭС. При этом основным механизмом упрочнения для ВЭС является твердорастворное упрочнение, наряду с которым возможно дополнительное упрочнение за счет формирования нанокристаллических упорядоченных структур, за счет формирования наноразмерных спинодальных структур. Все это дает возможность регулировать свойства ВЭС за счет введения микродобавок, позволяющих изменять микроструктуру сплава и механизм упрочнения, добиваясь наилучшего соотношения пластических и прочностных свойств.

Все ВЭС характеризуются прекрасной устойчивостью к рекристаллизации и разупрочнению при нагреве. Предполагается, что данные свойства обусловлены сравнительно низкой диффузией в ВЭС, обусловленной наличием большого числа атомов разнородных металлов. Результатом чего становится замедленные рост и укрупнение зерн в микроструктуре сплава при нагреве. Даже выше температуры начала рекристаллизации для ВЭС не наблюдается резкого падения показателей механических и прочностных свойств. Ряд ВЭС по своим магнитно-мягким свойствам сопоставим с ферритами. Сплавы ВЭС на основе системы $\mathrm{Co}-\mathrm{Cr}-\mathrm{Cu}-\mathrm{Fe}-\mathrm{Ni}$ по своим антикоррозионным свойствам не уступают коррозионностойким сталям. Тонкие пленки на основе системы $\mathrm{Al}-\mathrm{Co}-\mathrm{Cr}-\mathrm{Cu}-\mathrm{Fe}-\mathrm{Ni}$ по своим антиадгезионным свойствам близки к тефлону.

Исследования в области этих новых материалов можно условно разделить на три основных направления. Первое направление касается критериев формирования ВЭС. Второе направление адресуется к кристаллическому строению и микроструктуре и их взаимосвязи со свойствами материалов. Оба эти направления сравнительно хорошо изучены и развиты. Третье новое направление связано с разработкой ВЭС и созданием соответствующих баз данных в рамках CALPHAD метода [4]. Взаимосвязь между этими тремя направлениями исследований является невероятно важной. Критерии дают ответ на вопрос: «Где следует 
искать новые ВЭС» (новые компоненты, новые системы), позволяя исключить метод проб и ошибок при поиске новых материалов. Результаты второго направления, с одной стороны, позволяют проверить предложенные критерии. С другой стороны, эти результаты показывают, что эквиатомные ВЭС - это всего лишь отправная точка в дальнейшей разработке материалов. Для получения идеальной комбинации пластичности и прочности в будущем материале исходный эквиатомный ВЭС может быть изменен путем небольших модификаций его состава, что приводит к смене фаз или появлению новых фаз в его структуре и, как следствие, к изменениям в свойствах сплава. На этапе корректировки состава ВЭС, CAPHAD метод (третье направление) начинает играть главную роль. Следует подчеркнуть, что применение данного метода является весьма эффективным, так как позволяет прояснить характер фазовых превращений в сплавах, спрогнозировать типы и составы фаз, и таким образом, сократить число экспериментальных исследований. Создание термодинамических баз данных для ВЭС является востребованным современным направлением исследований.

Метастабильное расслоение жидкой фазы системы $\mathrm{Co}-\mathrm{Cr}-\mathrm{Cu}-\mathrm{Fe}-\mathrm{Ni}$, которое было экспериментально установлено в работах $[5,6]$, открывает возможность для создания новых композитных высокоентропийных материалов непосредственно из расплава. Эффективная разработка таких композитов, а также технологического процесса их получения нуждается в информации о термодинамических функциях смешения жидких эквиатомных сплавов. Таким образом, целью этой работы стало определение новых направлений и термодинамических подходов в разработке ВЭС в системе $\mathrm{Co}-\mathrm{Cr}-\mathrm{Cu}-\mathrm{Fe}-\mathrm{Ni}$.

Термодинамические свойства эквиатомных расплавов системы $\mathrm{Co}-\mathrm{Cr}-\mathrm{Cu}-\mathrm{Fe}-\mathrm{Ni}$ были рассчитаны с использованием собственной базы данных, которая включает в себя информацию о параметрах моделей, описывающих термодинамические функции смешения в десяти двухкомпонентных и десяти трехкомпонентных системах. Четыре двухкомпонентных и шесть тройных систем на основе переходных металлов, характеризуются стабильным или метастабильным расслоением жидкой фазы - $\mathrm{Co}-\mathrm{Cu}, \mathrm{Cu}-\mathrm{Fe}, \mathrm{Cu}-\mathrm{Ni}, \mathrm{Cr}-\mathrm{Cu}$ и $\mathrm{Co}-\mathrm{Cr}-\mathrm{Cu}, \mathrm{Co}-\mathrm{Cu}-\mathrm{Fe}, \mathrm{Co}-\mathrm{Cu}-\mathrm{Ni}$, $\mathrm{Cr}-\mathrm{Cu}-\mathrm{Fe}, \mathrm{Cr}-\mathrm{Cu}-\mathrm{Ni}, \mathrm{Cu}-\mathrm{Fe}-\mathrm{Ni}$. Bce термодинамические описания трехкомпонентных систем, представленные в базе данных и опирающиеся на общую базу параметров для граничных двухкомпонентных расплавов, являются, таким образом, самосогласованными.

Результаты расчета термодинамических функций смешения эквиатомных жидких сплавов системы $\mathrm{Co}-\mathrm{Cr}-\mathrm{Cu}-\mathrm{Fe}-\mathrm{Ni}$, проведенного для температур 1873 и $1500 \mathrm{~K}$, представлены в таблице 1 . В таблице также приведены идеальные составляющие энтропии $\left(\Delta_{\mathrm{m}} S^{\mathrm{id}}\right)$ и энергии Гиббса $\left(\Delta_{\mathrm{m}} G^{\mathrm{id}}\right)$ смешения эквиатомных расплавов при соответствующих температурах. Избыточные интегральные функции смешения четырехкомпонентных систем с медью и системы $\mathrm{Co}-\mathrm{Cr}-\mathrm{Cu}-\mathrm{Fe}-\mathrm{Ni}$ демонстрируют положительные отклонения от идеальности, что обусловлено положительными парными взаимодействиями медь-хром в системах $\mathrm{Co}-\mathrm{Cr}-\mathrm{Cu}$, $\mathrm{Cr}-\mathrm{Cu}-\mathrm{Ni}, \mathrm{Cr}-\mathrm{Cu}-\mathrm{Fe}$, медь-железо в системах $\mathrm{Cr}-\mathrm{Cu}-\mathrm{Fe}, \mathrm{Co}-\mathrm{Cu}-\mathrm{Fe}, \mathrm{Cu}-\mathrm{Fe}-\mathrm{Ni}$, медь-кобальт в системах $\mathrm{Co}-\mathrm{Cr}-\mathrm{Cu}, \mathrm{Co}-\mathrm{Cu}-\mathrm{Fe}$. Поведение компонентов в системе $\mathrm{Co}-\mathrm{Cr}-\mathrm{Fe}-\mathrm{Ni}$ близко к идеальному. Обращает на себя внимание то, что для четырехкомпонентных систем с медью подобные свойства для соответствующих температур оказываются близкими по значениям.

Избыточные термодинамические функции пятикомпонентного расплава мало отличаются от соответствующих значений для четырехкомпонентных жидких сплавов с медью. Энергия Гиббса смешения $\left(\Delta_{\mathrm{m}} G\right)$ эквиатомных жидких сплавов четырёхкомпонентных систем с медью и системы $\mathrm{Co}-\mathrm{Cr}-\mathrm{Cu}-\mathrm{Fe}-\mathrm{Ni}$ по абсолютному значению меньше, чем идеальная составляющая энергии Гиббса смешения. Можно отметить, что энергия Гиббса смешения пятикомпонентного расплава оказывается меньше аналогичной величины для четырехкомпонентных. Очевидной причиной этого является разница в $\Delta_{\mathrm{m}} G^{\mathrm{id}}$, характерная для этих систем. Обращает на себя внимание то, что в четырехкомпонентных системах с медью и в системе $\mathrm{Co}-\mathrm{Cr}-\mathrm{Cu}-\mathrm{Fe}-\mathrm{Ni}$ компенсации положительного вклада энтропийной составляющей в $\Delta_{\mathrm{m}} G$ любой из избыточных составляющих не происходит. 
Таблица 1

Термодинамические свойства жидких эквиатомных сплавов системы $\mathrm{Co}-\mathrm{Cr}-\mathrm{Cu}-\mathrm{Fe}-\mathrm{Ni}$

\begin{tabular}{|c|c|c|c|c|c|c|c|c|}
\hline Сплав & $T$ & $\Delta_{m} H$ & $\Delta_{\mathrm{m}} S^{\mathrm{ex}}$ & $\Delta_{\mathrm{m}} S^{\text {id }}$ & $\Delta_{\mathrm{m}} S$ & $\Delta_{\mathrm{m}} G^{e x}$ & $\Delta_{\mathrm{m}} G^{\text {id }}$ & $\Delta_{\mathrm{m}} G$ \\
\hline & $\mathrm{K}$ & кДж/моль & \multicolumn{3}{|c|}{ Дж/(моль К) } & \multicolumn{3}{|c|}{ кДж/моль } \\
\hline \multirow{2}{*}{$\mathrm{CoCrCuFe}$} & 1873 & 7,7 & 1,9 & \multirow{10}{*}{11,5} & 13,4 & 4,1 & $-21,6$ & $-17,5$ \\
\hline & 1500 & 8,3 & 2,3 & & 13,8 & 4,9 & $-17,3$ & $-12,4$ \\
\hline \multirow{2}{*}{$\mathrm{CoCrCuNi}$} & 1873 & 7,0 & 1,9 & & 13,4 & 3,4 & $-21,6$ & $-18,2$ \\
\hline & 1500 & 7,2 & 2,1 & & 13,6 & 4,1 & $-17,3$ & $-13,2$ \\
\hline \multirow{2}{*}{$\mathrm{CoCrFeNi}$} & 1873 & $-3,2$ & $-0,5$ & & 11,0 & $-2,3$ & $-21,6$ & $-23,9$ \\
\hline & 1500 & $-3,2$ & $-0,5$ & & 11,0 & $-2,5$ & $-17,3$ & $-19,8$ \\
\hline \multirow{2}{*}{$\mathrm{CoCuFeNi}$} & 1873 & 5,2 & 1,3 & & 12,8 & 2,7 & $-21,6$ & $-18,9$ \\
\hline & 1500 & 5,6 & 1,6 & & 13,1 & 3,3 & $-17,3$ & $-14,0$ \\
\hline \multirow{2}{*}{$\mathrm{CrCuFeNi}$} & 1873 & 5,6 & 1,4 & & 12,9 & 3,1 & $-21,6$ & $-18,5$ \\
\hline & 1500 & 6,2 & 1,7 & & 13,2 & 3,6 & $-17,3$ & $-13,7$ \\
\hline \multirow{2}{*}{$\mathrm{CoCrCuFeNi}$} & 1873 & 4,9 & 1,4 & \multirow{2}{*}{13,4} & 14,8 & 2,3 & $-25,1$ & $-22,8$ \\
\hline & 1500 & 5,2 & 1,6 & & 15,0 & 2,9 & $-20,1$ & $-17,2$ \\
\hline
\end{tabular}

Сопоставление значений термодинамических функций, рассчитанных при 1873 К и 1500 К, температуре при которой наблюдается стабильное расслоение жидких сплавов в системе $\mathrm{Cr}-\mathrm{Cu}-\mathrm{Fe}$, показали, что с понижением температуры положительные отклонения от идеальности избыточных термодинамических функций увеличились незначительно. Вместе со снижением температуры заметно уменьшается абсолютная величина идеального вклада в энергию Гиббса (таблица 1). Рост положительных значений избыточной энергии Гиббса смешения наряду с уменьшением абсолютных значений идеальной энергии Гиббса смешения с понижением температуры приводит к уменьшению термодинамической стабильности жидкой фазы, что указывает на склонность жидких эквиатомных сплавов к расслоению. На сегодняшнее время протекание расслоения жидкой фазы экспериментально установлено для эквиатомных сплавов систем $\mathrm{Co}-\mathrm{Cr}-\mathrm{Cu}-\mathrm{Fe}$ [7] и $\mathrm{Co}-\mathrm{Cr}-\mathrm{Cu}-\mathrm{Fe}-\mathrm{Ni}[5,6]$. Тот факт, что наибольшее значение функции $\Delta_{\mathrm{m}} G$ соответствует эквиатомному сплаву системы $\mathrm{Co}-\mathrm{Cr}-\mathrm{Cu}-\mathrm{Fe}$, качественно согласуется со стабильным расслоением именно ее расплавов. В системе $\mathrm{Co}-\mathrm{Cr}-\mathrm{Cu}-\mathrm{Fe}-\mathrm{Ni}$ наблюдаются наиболее слабые положительные отклонения от идеальности и низкие значения функции $\Delta_{\mathrm{m}} G$ для эквиатомного расплава, что указывает на его повышенную термодинамическую стабильность по сравнению с эквиатомным жидким сплавом системы $\mathrm{Co}-\mathrm{Cr}-\mathrm{Cu}-\mathrm{Fe}$. В результате такого совместного действия указанных выше факторов, расслоение в системе $\mathrm{Co}-\mathrm{Cr}-\mathrm{Cu}-\mathrm{Fe}-\mathrm{Ni}$ имеет метастабильный характер.

Фазовые превращения в системе $\mathrm{Co}-\mathrm{Cr}-\mathrm{Cu}-\mathrm{Fe}-\mathrm{Ni}$ и ее подсистемах представляют возможность для выбора составов дисперсно-упрочняемых ВЭС с наиболее высокой предельной рабочей температурой и соответствующих параметров термической или термомеханической обработки [8-10]. Поскольку экспериментальное исследование пятикомпонентной пространства в координатах состав-температура требует слишком много времени, затрат и усилий, оно должно быть дополнено компьютерным моделированием фазовых превращений в системе. Одной из наиболее успешных методик в области компьютерного моделирования фазовых равновесий является CALPHAD-метод, в рамках которого могут быть рассчитаны температурно-концентрационные границы существования род-ких и кристаллических фаз с помощью термодинамических баз данных [4].

Для понимания характера фазовых равновесий в пятикомпонентной системе необходим анализ фазовых превращений в ограничивающих четырехкомпонентных системах. Результаты моделирования показали, что по характеру фазовых равновесий можно условно выделить две группы систем - четыре системы с медью (Co- $\mathrm{Cr}-\mathrm{Cu}-\mathrm{Fe}, \mathrm{Co}-\mathrm{Cr}-\mathrm{Cu}-\mathrm{Ni}$, $\mathrm{Co}-\mathrm{Cu}-\mathrm{Fe}-\mathrm{Ni}, \mathrm{Cr}-\mathrm{Cu}-\mathrm{Fe}-\mathrm{Ni}$ ), для которых характерно распад ГЦК раствора на ГЦК богатый медью, и ГЦК с высоким содержанием хрома, кобальта, железа и никеля, и одна система на основе хрома и триады железа, в которой наблюдается высокая взаимная растворимость компонентов. Согласно нашим расчетам температуры распада ГЦК раствора 
на ГЦК и ГЦК 2 фазы в четырехкомпонентных системах с медью выше, чем в трехкомпонентных системах, а эквиатомные и околоэквиатомные сплавы являются двухфазными. Такое поведение связано с тем, что для систем с медью наблюдается смещение минимума энергии Гиббса смешения ГЦК раствора до предельных систем $\mathrm{Fe}-(\mathrm{Cr}, \mathrm{Co}, \mathrm{Ni})$. Bследствие этого, ГЦК раствор в эквиатомных трехкомпонентных сплавах оказывается менее термодинамически стабильным, чем в трехкомпонентных сплавах, близких к $\mathrm{Fe}-(\mathrm{Cr}, \mathrm{Co}, \mathrm{Ni})$. Отличительной чертой системы $\mathrm{Co}-\mathrm{Cr}-\mathrm{Fe}-\mathrm{Ni}$ является образование протяженных областей гомогенности для ОЦК и ГЦК растворов. Согласно нашим расчетам, введение хрома в сплавы на основе триады железа приводит к стабилизации ОЦК раствора в широкой концентрационной области, железо и никель способствуют формированию ГЦК раствора, при повышенном содержании кобальта наблюдается распад ГЦК раствора на ферромагнитные и парамагнитные ГЦК структуры, при высоком содержании железа в сплавах выпадает ОЦК фаза.

Характер фазовых превращений является близким в четырехкомпонентных системах с медью и в системе $\mathrm{Co}-\mathrm{Cr}-\mathrm{Cu}-\mathrm{Fe}-\mathrm{Ni}$. Согласно нашим расчетам эквиатомный сплав $\mathrm{Co}-\mathrm{Cr}-\mathrm{Cu}-\mathrm{Fe}-\mathrm{Ni}$ является двухфазным со структурой ГЦК+ОЦК и не может быть подвергнут термической обработке для получения дисперсионного упрочнения, поскольку отсутствует температурный интервал, в котором данный сплав имеет однофазную кристаллическую структуру.

\section{ВЫВОДЫ}

Анализируя влияние каждого из компонентов на характер фазовых равновесий в системе $\mathrm{Co}-\mathrm{Cr}-\mathrm{Cu}-\mathrm{Fe}-\mathrm{Ni}$, можно прийти к следующим выводам. Элементы триады железа, особенно никель, способствуют стабилизации ГЦК раствора. Высокое содержание хрома и железа стабилизируют ОЦК фазу в сплавах. Высокое содержание меди в пятикомпонентной сплавах приводит к формированию двухфазной структуры. Исходя из выше сказанного можно предположить, что перспективные составы дисперсно-упрочненных ВЭС будут отвечать концентрационной области со сравнительно высоким содержанием никеля. Концентрация меди в сплавах не должна превышать 5-10 т.\%. Уменьшение содержания хрома и увеличение содержания никеля приводит к расширению области гомогенности ГЦК 1 фазы. Концентрации кобальта и железа в ВЭС должны быть выше содержания хрома, но меньше содержания никеля для получения температурного интервала, в котором данный сплав имеет однофазную кристаллическую структуру.

\section{СПИСОК ИСПОЛЬЗОВАННОЙ ЛИТЕРАТУРЫ}

1. US 2002/0159917 A1, Int. Cl. C22C 30/00. High-entropy multielemental alloys / J.-W. Yeh // No. 09/706,710; appl. Apr. 29, 2002; pub. Oct. 31. 2002, Patent publication application.

2. Murty B. S., High-entropy alloys / B.S. Murty, J.W. Yeh, S. Ranganathan // $1^{\text {st }}$ Ed. - ButterworthHeinemann, London, 2014. - 218 pp.

3. Zhang Y. Microstructures and properties of high-entropy alloys / Y. Zhang, T.T. Zuo, Z. Tang et al. // Progr. Mater. Sci. - 2014. - V. 61. - P. 1-93.

4. Saunders N., Miodownik A. P. CALPHAD (Calculation of Phase Diagrams): A Comprehensive Guide, Vol. 1. - Pergamon, Oxford : 1998. - 478 p.

5. Wu P. H. Microstructures and liquid phase separation in multicomponent CoCrCuFeNi high entropy alloys / P. H. Wu, N. Liu, P. J. Zhou [et al] // Mater. Sci. Tech. - 2016. - V. 32, No. 6. - P. 576-580.

6. Wang W.L. Liquid phase separation and rapid dendritic growth of high-entropy CoCrCuFeNi alloy / W. L. Wang, L. Hu, S. B. Luo [et al] // Intermetallics. - 2016. - V. 77. - P. 41-45.

7. Munitz A., Liquid phase separation in transition element high entropy alloys / A. Munitz, M. J. Kaufman, R. Abbaschian // Intermetallics. - 2017. - V. 86. - P. 59-72.

8. Senkov O. N. Accelerated exploration of multi-principal element alloys for structural applications / O. Senkov, J. Miller, D. Miracle, C. Woodward // Calphad. - 2015. - Vol. 50. - P. 32-48.

9. Tong C.-J. Microstructure characterization of $\mathrm{Al}_{x} \mathrm{CoCrCuFeNi}$ high-entropy alloy system with multiprincipal elements / C.-J. Tong, Y.-L. Chen, J.-W. Yeh [et al] // Metall. Mater. Trans. A. - 2005. - Vol. 36, № 4. - P. $881-893$.

10. Wang X. F. Novel microstructure and properties of multicomponent CoCrCuFeNiTi $i_{x}$ alloys / X. Wang, Y. Zhang, Y. Qiao, G. Chen // Intermetallics. - 2007. - Vol. 15, № 3. - P. 357-362. 